\title{
TSC2 Mutations Were Associated with the Early Recurrence of Patients with HCC Underwent Hepatectomy
}

\section{Kangjian Song $(\mathbb{D}$ \\ Fu $\mathrm{He}$ \\ Yang Xin \\ Ge Guan (ID) \\ Junyu Huo (D) \\ Qingwei Zhu \\ Ning Fan \\ Yuan Guo \\ Yunjin Zang \\ Liqun $\mathrm{Wu}$}

Liver Disease Center, The Affiliated Hospital of Qingdao University, Qingdao, 266003, People's Republic of China
Correspondence: Liqun Wu

Liver Disease Center, The Affiliated Hospital of Qingdao University, No. 59 Haier Road, Laoshan District, Qingdao, Shandong, 266003, People's Republic of China

Tel +86 I53I532833I

Email wulq58I0@I26.com
This article was published in the following Dove Press journal: Pharmacogenomics and Personalized Medicine

Purpose: To explore the value of Tuberous sclerosis complex 2 (TSC2) mutations in evaluating the early recurrence of hepatocellular carcinoma (HCC) patients underwent hepatectomy.

Patients and Methods: A total of 183 HCC patients were enrolled. Next-generation sequencing was performed on tumor tissues to analyze genomic alterations, tumor mutational burden and variant allele fraction (VAF). The associations between TSC2 mutations and recurrence rate within 1 year, RFS and OS after hepatectomy were analyzed.

Results: Our results showed that TSC2 mutation frequency in HCC was $12.6 \%$. Compared to patients without TSC2 mutation, the proportion of microvascular invasion (MVI) and Edmondson grade III-IV was significantly higher in patients with a TSC2 mutation $(\mathrm{p}<0.05)$. The VAF of mutated TSC 2 was higher in patients with maximum diameter of tumor $>5 \mathrm{~cm}$ or MVI than that of other patients $(\mathrm{p}<0.05)$. The frequency of TP53 mutation was significantly higher in patients with a TSC2 mutation than those without TSC2 mutation ( $\mathrm{p}=0.003$ ). Follow-up analysis showed that patients with a TSC2 mutation had significantly higher recurrence rate within 1 year $(p=0.015)$ and poorer median recurrence-free survival (RFS) $(p=0.010)$ than patients without TSC2 mutation. TSC2 mutations did not significantly affect overall survival of patients $(p=0.480)$. The multivariate analysis results showed that the Barcelona Clinic Liver Cancer (BCLC) B-C stage, TSC2 mutations and preoperative serum alpha-fetoprotein level $\geq 400 \mu \mathrm{g} / \mathrm{L}$ were independently associated with recurrence within 1 year after hepatectomy $(\mathrm{HR}=8.628,95 \%$ CI: 3.836-19.405, $p=0.000 ; \mathrm{HR}=3.885,95 \%$ CI: $1.295-11.653$, $p=0.015$; HR $=2.327,95 \% \mathrm{CI}: 1.018-5.323, p=0.045$; respectively), and poorer $\mathrm{RFS}$ after hepatectomy $(\mathrm{HR}=3.070,95 \% \mathrm{CI}: 1.971-4.783, p=0.000 ; \mathrm{HR}=1.861,95 \% \mathrm{CI}$ : 1.061-3.267, $p=0.030$; HR=1.715, 95\% CI: 1.093-2.693, $p=0.019$; respectively).

Conclusion: TSC2 mutations were significantly associated with MVI in liver paracarcinoma tissue and Edmondson grade III-IV in patients with HCC and were independently associated with recurrence within 1 year and poorer RFS after hepatectomy. The TSC2 mutation may be a potential predictor for early recurrence in $\mathrm{HCC}$ patients underwent hepatectomy.

Keywords: hepatocellular carcinoma, tuberous sclerosis complex 2, next-generation sequencing, gene mutation, early recurrence

\section{Introduction}

Hepatocellular carcinoma (HCC) is the sixth most common cancer and the fourth most common cause of cancer-related death worldwide. ${ }^{1}$ Surgery is the main 
treatment for HCC patients, including liver transplantation, liver resection and ablation. However, the risk of recurrence after surgical treatment is high. ${ }^{2,3}$

Tuberous sclerosis complex 2 (TSC2) is an important tumor-suppressor gene, which was firstly found in tuberous sclerosis complex. ${ }^{4}$ Many studies have demonstrated that TSC2 closely related with several cancers. For example, Mehta et $\mathrm{al}^{5}$ reported that the expression of TSC2 was downregulated in aggressive breast cancer. Chakraborty et al $^{6}$ found that the methyltransferase inhibitor 5-azacytidine could significantly increase the expression of TSC2 in oral squamous cell carcinoma cell lines. In a prognostic model for lung adenocarcinoma established by Geng et al, ${ }^{7}$ TSC2 was a biomarker to predict a poor prognosis. Lee et $\mathrm{al}^{8}$ also reported that TSC2 rs30259G > A mutation could predict shorter OS and DFS of non-small cell lung cancer patients after curative surgery.

Currently, some studies have reported that TSC2 could be a therapeutic target in HCC. ${ }^{9-11}$ However, the value of TSC2 in predicting the prognosis after hepatectomy was rarely reported. In this study, we aimed to detect the genomic variations (GAs) of $\mathrm{HCC}$ and evaluated the potential value of $T S C 2$ in predicting the prognosis of HCC patients after hepatectomy.

\section{Patients and Methods}

\section{Patients}

A total of 183 HCC patients who were treated by hepatectomy at the Affiliated Hospital of Qingdao University from March 2017 to February 2020 were enrolled in this study and no extrahepatic metastasis was found in all patients before surgery. Among the enrolled patients, 161 patients were infected by hepatitis $B$ virus (HBV), while those infected by hepatitis $C$ virus and underwent antitumor therapy before liver resection were excluded. The surgical margins of all patients were achieved R0. The preoperative serological results and clinicopathological characteristics were shown in Table 1.

\section{Identification of Genetic Alterations, $\mathrm{TMB}$, and $\mathrm{VAF}$}

Formalin-fixed, paraffin-embedded (FFPE) tissues were collected from patients for next-generation sequencing (NGS). The genes were captured and sequenced by genomic profile produced using the NGS-based YuanSu 450 gene panel. Genetic alterations (GAs) were identified as follows: single nucleotide variants (SNVs) were identified
Table I Clinicopathological Characteristics of HCC Patients

\begin{tabular}{|c|c|}
\hline Clinicopathological Characteristics & Number of Patients \\
\hline Age $(<65 / \geq 65)$ & $144 / 39$ \\
\hline Gender (male/female) & $156 / 27$ \\
\hline Hypertension (no/yes) & $137 / 46$ \\
\hline Diabetes (no/yes) & $162 / 2 \mid$ \\
\hline Family history of cancer (no/yes) & $128 / 55$ \\
\hline History of alcoholism (no/yes) & $116 / 67$ \\
\hline HBsAg (negative/positive) & $22 / 161$ \\
\hline HBV-DNA $(<|E+003 / \geq| E+003 \mid \mathrm{I} / \mathrm{mL})$ & $126 / 57$ \\
\hline Anti-hepatitis virus treatment (no/yes) & $103 / 80$ \\
\hline $\operatorname{AFP}(<400 / \geq 400 \mu \mathrm{g} / \mathrm{L})$ & $130 / 53$ \\
\hline Tumor number (single/multiple) & $130 / 53$ \\
\hline Tumor size $(\leq 5 \mathrm{~cm} />5 \mathrm{~cm})$ & $118 / 65$ \\
\hline BCLC (0-A/B-C) & $127 / 56$ \\
\hline Macrovascular invasion (no/yes) & $163 / 20$ \\
\hline Edmondson grade (I-II/III-IV) & $93 / 90$ \\
\hline MVI (no/yes) & $93 / 90$ \\
\hline
\end{tabular}

by MuTect (v1.7); Insertion-deletions (InDels) were identified by using PINDEL (V0.2.5). The functional impact of GAs was annotated by SnpEff3.0. Copy number variations (CNV) regions were identified by Control-FREEC (v9.7). Gene rearrangement/fusion was detected through an inhouse developed pipeline. Tumor mutational burden (TMB) was estimated by counting the coding somatic mutations, including SNVs and Indels, per megabase of the sequence examined in each patient. Variant allele fraction (VAF) was calculated by dividing the number of mutated bases by the total base number of the site. The concept of VAF was only for SNVs and short InDels due to biological information algorithms. Thus, there was no VAF on the CNV or gene rearrangement/fusion.

\section{Follow-Up}

All patients enrolled in this study were followed up regularly after surgery. During the first 3 months after liver resection, the patients were followed up once a month; during 3-24 months after liver resection, they were followed up every 3 months; and after 2 years, they were followed up every 6 months. The follow-up examination 
included serum alpha-fetoprotein (AFP), liver function, ultrasonic examination of liver and computed tomography of lung. Patients received the contrast-enhanced computed tomography (CT) scan of upper abdomen annually. When suspected signs of recurrence were found, contrastenhanced CT or magnetic resonance imaging (MRI) was performed to clarify the diagnosis. Recurrence-free survival (RFS) was confirmed by imaging examination. The patients were followed up until August 312020 or died.

\section{Statistical Analysis}

Statistical analysis was performed using SPSS 22.0 (IBM). Kaplan-Meier curves were drawn using GraphPad Prism 7.0. Chi-square test or Fisher's exact test was used for qualitative data in univariate analysis and logistic regression was used for multivariate analysis. Mann-Whitney $U$-test was used to analyze the correlation between VAF of TSC2 and clinicopathological characteristics. KaplanMeier curve analysis and Log rank test were used to compare RFS and OS in different groups. Variables associated with RFS were assessed by Cox regression model and variables with $p$ values $<0.05$ in univariate analysis were subjected to multivariate analysis. $P<0.05$ was considered to be statistically significant.

\section{Results}

\section{Baseline Data of HCC Patients}

In this cohort, a total of 183 HCC patients were enrolled. The main characteristics of patients were shown in Table 1. Among them, there were 161 patients with serum hepatitis B surface antigen ( $\mathrm{HBsAg}$ ) positive, 53 patients with preoperative serum AFP level above $400 \mu \mathrm{g} / \mathrm{L}$ and 20 patients with macrovascular invasion. The BCLC stage of 22, 105, 36, 20 patients was $0, \mathrm{~A}, \mathrm{~B}$ and C, respectively. In pathological results, the Edmondson grades of tumors were I-II ( $\mathrm{n}=93)$ and III-IV ( $\mathrm{n}=90)$, and the MVI were found in liver para-carcinoma tissues of 90 patients.

\section{The Correlation Between TSC2}

\section{Mutations and Clinicopathological}

\section{Characteristics}

Out of 183 specimens, 23 (12.6\%) were harboring TSC2 mutations, including $15 \mathrm{SNVs}, 7 \mathrm{InDels}$, and $1 \mathrm{CNV}$ (Table 2). Compared to patients without TSC2 mutation, the proportion of MVI and Edmondson grade III-IV was significantly higher in patients with a TSC2 mutation ( $p=0.011$ and $p=0.036$, respectively) (Table 3 ). We did
Table 2 Alterations of TSC2 in 23 Patients

\begin{tabular}{|c|c|c|c|}
\hline Patients & Alteration Type & Coding DNA Change & VAF \\
\hline I & SNV & $139-2 A>G$ & 0.31 \\
\hline 2 & SNV & 2299del & 0.29 \\
\hline 3 & SNV & $1906 \mathrm{G}>\mathrm{T}$ & 0.08 \\
\hline 4 & SNV & $319 \mathrm{G}>\mathrm{A}$ & 0.12 \\
\hline 5 & SNV & $849-2 A>C$ & 0.12 \\
\hline 6 & SNV & 3496del & 0.19 \\
\hline 7 & SNV & I643del & 0.15 \\
\hline 8 & InDel & 1716+1904_3035del & 0.49 \\
\hline 9 & SNV & $337-2 A>C$ & 0.20 \\
\hline 10 & SNV & $4037 C>A$ & 0.44 \\
\hline 11 & InDel & II0_139-344del & 0.22 \\
\hline 12 & $\mathrm{CNV}$ & Gene deletion & - \\
\hline 13 & InDel & 3560_356Idel & 0.07 \\
\hline 14 & SNV & $5 \mid 38 \mathrm{G}>\mathrm{A}$ & 0.63 \\
\hline 15 & InDel & $|7| 7-|2| \_\mid 840-167 \mathrm{del}$ & 0.97 \\
\hline 16 & InDel & $1362-133 \_1716+507 \mathrm{del}$ & 0.11 \\
\hline 17 & SNV & $648+1 G>T$ & 0.29 \\
\hline 18 & SNV & 365I_3652insA & 0.25 \\
\hline 19 & InDel & exon2_exon3del & - \\
\hline 20 & SNV & $65 G>C$ & 0.01 \\
\hline 21 & SNV & $1257+2 T>A$ & 0.09 \\
\hline 22 & InDel & 1444-235_1665del & 0.13 \\
\hline 23 & SNV & $2242 \mathrm{G}>\mathrm{T}$ & 0.26 \\
\hline
\end{tabular}

not find a significant association between TSC2 mutations and other clinicopathological characteristics (Table 3).

\section{The Correlation Between VAF of TSC2 Mutations and Clinicopathological Characteristics}

In the subgroup with TSC2 mutations, VAF of mutated TSC 2 could be calculated in 21 cases. The median VAF was 0.20 (range, 0.01-0.97). By Mann-Whitney $U$-test, we found VAF of mutated TSC2 was associated with MVI and tumor size. The VAF of mutated TSC2 was significantly higher in patients with MVI and maximum diameter of tumor $>5 \mathrm{~cm}(p<0.05)$ (Table 4$)$. 
Table 3 The Correlation Between TSC2 Mutations and Clinicopathological Characteristics

\begin{tabular}{|c|c|c|c|c|}
\hline \multirow{2}{*}{$\begin{array}{l}\text { Clinicopathological } \\
\text { Characteristics }\end{array}$} & \multicolumn{4}{|c|}{ TSC2 } \\
\hline & $\begin{array}{l}\text { Wild } \\
\text { Type }\end{array}$ & Mutant & $\chi^{2}$ & $\mathbf{P}$ \\
\hline Age $(<65 / \geq 65)$ & $125 / 35$ & $19 / 4$ & - & 0.788 \\
\hline Gender (male/female) & $138 / 22$ & $18 / 5$ & - & 0.345 \\
\hline Hypertension (no/yes) & $116 / 44$ & $21 / 2$ & 3.779 & 0.052 \\
\hline Diabetes (no/yes) & $141 / 19$ & $21 / 2$ & - & 1.000 \\
\hline $\begin{array}{l}\text { Family history of cancer (nol } \\
\text { yes) }\end{array}$ & $111 / 49$ & $17 / 6$ & 0.197 & 0.657 \\
\hline $\begin{array}{l}\text { History of alcoholism (no/ } \\
\text { yes) }\end{array}$ & $99 / 61$ & $17 / 6$ & 1.256 & 0.262 \\
\hline HBsAg (negative/positive) & $22 / 138$ & $0 / 23$ & - & 0.081 \\
\hline $\operatorname{AFP}(<400 / \geq 400 \mu \mathrm{g} / \mathrm{L})$ & $115 / 45$ & $15 / 8$ & 0.433 & 0.510 \\
\hline $\begin{array}{l}\text { Tumor number (single/ } \\
\text { multiple) }\end{array}$ & $115 / 45$ & $15 / 8$ & 0.433 & 0.510 \\
\hline Tumor size $(\leq 5 \mathrm{~cm} />5 \mathrm{~cm})$ & $102 / 58$ & $16 / 7$ & 0.297 & 0.586 \\
\hline$B C L C(0-A / B-C)$ & $112 / 48$ & $15 / 8$ & 0.217 & 0.642 \\
\hline $\begin{array}{l}\text { Macrovascular invasion (no/ } \\
\text { yes) }\end{array}$ & $143 / 17$ & $20 / 3$ & - & 0.722 \\
\hline Edmondson grade (I-II/III-IV) & $86 / 74$ & $7 / 16$ & 4.374 & 0.036 \\
\hline MVI (no/yes) & $87 / 73$ & $6 / 17$ & 6.438 & 0.011 \\
\hline
\end{tabular}

Table 4 The Correlation Between VAF of TSC2 Mutations and Clinicopathological Characteristics

\begin{tabular}{|c|c|c|c|}
\hline \multirow{2}{*}{$\begin{array}{l}\text { Clinicopathological } \\
\text { Characteristics }\end{array}$} & \multicolumn{3}{|c|}{ VAF of TSC2 } \\
\hline & Median & $\boldsymbol{U}$ & $\mathbf{P}$ \\
\hline Age $(<65 / \geq 65)$ & $0.220 / 0.195$ & 48.500 & 0.654 \\
\hline Gender (male/female) & $0.210 / 0.130$ & 25.500 & 0.240 \\
\hline Family history of cancer (no/yes) & $0.190 / 0.255$ & 29.000 & 0.698 \\
\hline History of alcoholism (no/yes) & $0.200 / 0.200$ & 42.500 & 0.850 \\
\hline $\operatorname{AFP}(<400 / \geq 400 \mu \mathrm{g} / \mathrm{L})$ & $0.225 / 0.130$ & 38.000 & 0.443 \\
\hline Tumor number (single/multiple) & $0.170 / 0.220$ & 35.500 & 0.322 \\
\hline Tumor size $(\leq 5 \mathrm{~cm} />5 \mathrm{~cm})$ & $0.150 / 0.400$ & 19.000 & 0.045 \\
\hline BCLC (0-A/B-C) & $0.170 / 0.220$ & 35.500 & 0.322 \\
\hline Edmondson grade (I-II/III-IV) & $0.235 / 0.190$ & 44.500 & 0.970 \\
\hline MVI (no/yes) & $0.080 / 0.235$ & 13.000 & 0.025 \\
\hline
\end{tabular}

\section{The Correlation Between TSC2}

\section{Mutations and Other Genes}

The most commonly mutated genes of enrolled patients were TP53 (54.1\%), TERT (41.0\%), CTNNB1 (23.0\%), AXIN1 (14.8\%) and TSC2 (12.6\%). The mutated mTOR pathway-related genes were TSC2 $(\mathrm{n}=23), \operatorname{PTEN}(\mathrm{n}=7)$, TSCl $(\mathrm{n}=5), \operatorname{mTOR}(\mathrm{n}=5), P I K 3 C A(\mathrm{n}=3), N F 1(\mathrm{n}=3)$, STK11 (n=3), AKT2 (n=2). This result demonstrated that TSC2 gene was the most frequently mutant gene among mTOR pathway-related genes in our study.

In this study, we found that co-mutations between TSC2 and TP53 were detected in 19 patients, 9 patients had co-mutations between TSC 2 and TERT, 3 patients had co-mutations between $T S C 2$ and $C T N N B 1,5$ patients had comutations between TSC2 and AXIN1. We also found TSC1 mutations in 5 patients and no patient had co-mutation of TSC2 and TSC1. Univariate analysis identified the correlation between TSC2 mutations and TP53 mutations. Compared to patients without a TSC2 mutation, the proportion of patients with a TP53 mutation was significantly higher in patients with a TSC2 mutation $(p=0.003)$ (Table 5).

TMB values were calculated in all 183 HCC specimens, and the $75 \%$ TMB threshold value was 8.5 mutations $/ \mathrm{Mb}$. TMB value higher than 8.5 mutations/Mb was defined as TMB-H, and those lower than 8.5 mutations/Mb was defined as TMB-L. The patients with a TSC2 mutation was account for $13.5 \%$ in the TMB-L group, while was account for $10.0 \%$ in the TMB-H group. There was no correlation between TMB and TSC2 mutations ( $p=0.520$ ) (Table 5).

\section{Follow-Up Results Analysis HCC Recurrence}

The median follow-up time of 183 patients was 15.5 months (range, 4.6-40.7 months). The recurrence was found in 87

Table 5 The Correlation Between TSC2 Mutations and Other Genes

\begin{tabular}{|l|c|c|c|c|}
\hline \multirow{2}{*}{ Other Mutant Genes } & \multicolumn{4}{|c|}{ TSC2 } \\
\cline { 2 - 5 } & $\begin{array}{l}\text { Wild } \\
\text { Type }\end{array}$ & Mutant & $\chi^{2}$ & $P$ \\
\hline TP53 (Wild type/Mutant) & $80 / 80$ & $4 / 19$ & 8.611 & 0.003 \\
\hline TERT (Wild type/Mutant) & $94 / 66$ & $14 / 9$ & 0.037 & 0.847 \\
\hline CTNNBI (Wild type/Mutant) & $121 / 39$ & $20 / 3$ & 1.460 & 0.227 \\
\hline AXINI (Wild type/Mutant) & $138 / 22$ & $18 / 5$ & - & 0.345 \\
\hline TMB (<8.5/ $\geq 8.5$ mutations/Mb) & $115 / 45$ & $18 / 5$ & 0.413 & 0.520 \\
\hline
\end{tabular}


Table 6 The Correlation Between Different Factors and Recurrence Within I Year After Hepatectomy

\begin{tabular}{|c|c|c|c|c|c|c|}
\hline \multirow[t]{2}{*}{ Variables } & \multicolumn{3}{|c|}{ Univariate Analysis } & \multicolumn{3}{|c|}{ Multivariate Analysis } \\
\hline & $\mathbf{n} / \mathbf{n}$ & $\chi^{2}$ & $\mathbf{P}$ & HR & $95 \% \mathrm{Cl}$ & $\mathbf{P}$ \\
\hline Age $(<65 / \geq 65)$ & $46 / 11$ & 0.202 & 0.653 & & & \\
\hline Gender (male/female) & $50 / 7$ & 0.316 & 0.574 & & & \\
\hline Hypertension (no/yes) & $49 / 8$ & 3.638 & 0.056 & & & \\
\hline Diabetes (no/yes) & $53 / 4$ & 1.213 & $0.27 \mathrm{I}$ & & & \\
\hline Family history of cancer (no/yes) & $42 / 15$ & 0.774 & 0.379 & & & \\
\hline History of alcoholism (no/yes) & $36 / 21$ & 0.000 & 0.995 & & & \\
\hline HBsAg (negative/positive) & $3 / 54$ & 3.179 & 0.075 & & & \\
\hline HBV-DNA $(<|E+003 / \geq| E+003 \mid \mathrm{U} / \mathrm{mL})$ & $35 / 22$ & 1.197 & 0.274 & & & \\
\hline Anti-hepatitis virus treatment (no/yes) & $34 / 23$ & 0.167 & 0.682 & & & \\
\hline $\operatorname{AFP}(<400 / \geq 400 \mu \mathrm{g} / \mathrm{L})$ & $32 / 25$ & 10.844 & 0.001 & 2.327 & $1.018-5.323$ & 0.045 \\
\hline$B C L C(0-A / B-C)$ & $23 / 34$ & 37.065 & 0.000 & 8.628 & $3.836-19.405$ & 0.000 \\
\hline Liver fibrosis (SIS2/S3S4) & $|4 / 4|$ & 0.277 & 0.598 & & & \\
\hline Edmondson grade (I-II/III-IV) & $24 / 33$ & 4.318 & 0.038 & - & - & 0.933 \\
\hline MVI (no/yes) & $19 / 38$ & 11.376 & 0.001 & - & - & 0.161 \\
\hline TP53 (Wild type/Mutant) & $23 / 34$ & 0.993 & 0.319 & & & \\
\hline TERT (Wild type/Mutant) & $31 / 26$ & 0.914 & 0.339 & & & \\
\hline CTNNBI (Wild type/Mutant) & $45 / 12$ & 0.106 & 0.744 & & & \\
\hline AXINI (Wild type/Mutant) & $50 / 7$ & 0.161 & 0.688 & & & \\
\hline TSC2 (Wild type/Mutant) & $45 / 12$ & 5.922 & 0.015 & 3.885 & $1.295-11.653$ & 0.015 \\
\hline TMB (<8.5/ $\geq 8.5$ mutations/Mb) & $45 / 12$ & 0.035 & 0.852 & & & \\
\hline
\end{tabular}

patients. Among our cohort, 160 patients were followed up for more than 1 year and 57 patients of them were found with HCC recurrence within 1 year after surgery. By Chi-square test, the results showed that the recurrence rate within 1 year in patients with a TSC2 mutation was significantly higher than patients without a TSC2 mutation $(60 \%$ vs $32 \%$, $p=0.015$ ) (Table 6). Besides, some other clinicopathological factors were significantly associated with recurrence within 1 year, including serum AFP, BCLC stage, Edmondson grade and MVI $(p<0.05)$ (Table 6). By logistic regression analysis, we found BCLC B-C stage $(\mathrm{HR}=8.628$, 95\% CI: 3.836-19.405, $p=0.000)$, TSC2 mutations ( $\mathrm{HR}=3.885$, 95\% CI: 1.295-11.653, $\quad p=0.015)$ and serum AFP $\geq 400 \mu \mathrm{g} / \mathrm{L}$ $(\mathrm{HR}=2.327,95 \% \mathrm{CI}: 1.018-5.323, p=0.045)$ were independently associated with recurrence within 1 year after surgery (Table 6).

\section{RFS and OS}

By Kaplan-Meier analysis, the median RFS of patients with a TSC2 mutation was 7.4 months, while the median RFS of patients without a TSC2 mutation was 30.8 months. Patients with a TSC2 mutation had significantly poorer RFS than patients without a TSC2 mutation $(p=0.010)$ (Figure 1). However, TSC2 mutations did not significantly affect overall survival of patients $(p=0.480)$ (Figure 2).

In univariate analysis, we found some factors which were significantly correlated with RFS, including TSC2 mutations, BCLC stage, MVI, Edmondson grade, serum AFP and hypertension $(p<0.05)$ (Table 7). By multivariate Cox regression analysis, the results showed that BCLC $\mathrm{B}-\mathrm{C}$ stage $(\mathrm{HR}=3.070,95 \% \mathrm{CI}: 1.971-4.783, p=0.000)$, TSC2 mutation $(\mathrm{HR}=1.861,95 \% \quad \mathrm{CI}:$ 1.061-3.267, 


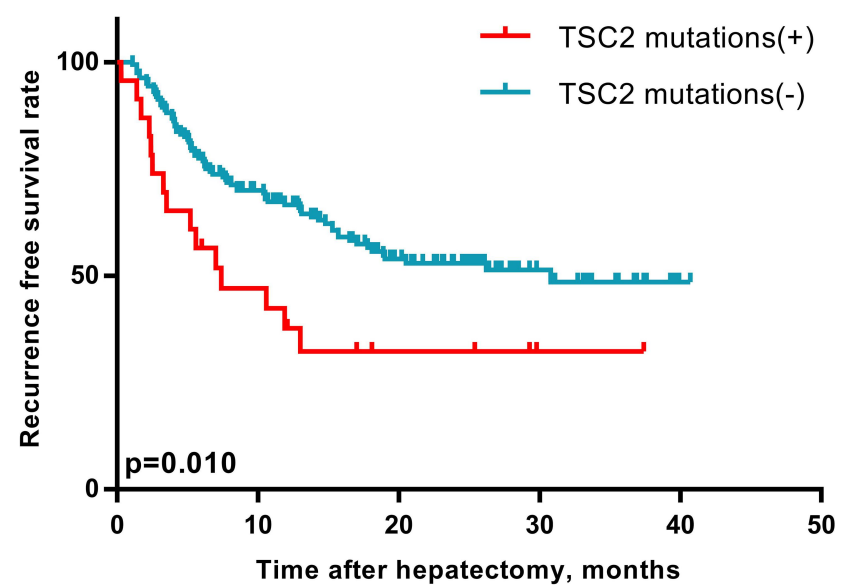

Figure I Survival curves of RFS according to TSC2 mutational status evaluated by Kaplan-Meier method $(n=183)$. There were 23 patients with a TSC2 mutation and 160 patients without TSC2 mutation. The median RFS of patients with a TSC2 mutation was 7.4 months, while the median RFS of patients without TSC2 mutation was 30.8 months. Patients with a TSC2 mutation had significantly poorer RFS than others $(p=0.010)$.

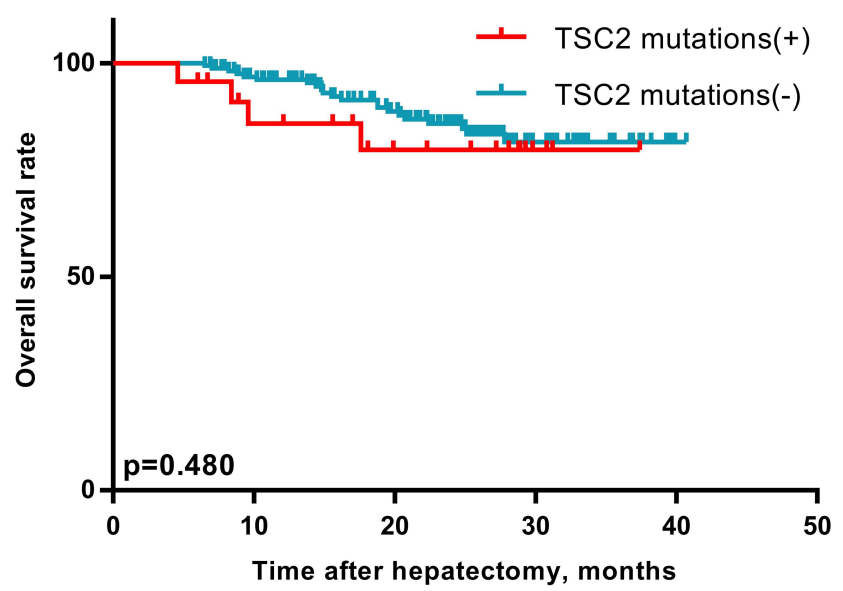

Figure 2 Survival curves of OS according to TSC2 mutational status evaluated by Kaplan-Meier method $(n=183)$. There were 23 patients with a TSC2 mutation and 160 patients without TSC2 mutation. TSC2 mutations did not significantly affect overall survival of patients $(p=0.480)$.

$p=0.030)$ and preoperative serum AFP level $\geq 400 \mu \mathrm{g} / \mathrm{L}$ $(\mathrm{HR}=1.715,95 \%$ CI: 1.093-2.693, $p=0.019)$ were independent risk factors for poor RFS in HCC patients after hepatectomy (Table 7).

There were 8 patients with a TSC2 mutation and 45 patients without TSC2 mutation on the patients with serum AFP $\geq 400 \mu \mathrm{g} / \mathrm{L}$ group $(\mathrm{n}=53)$. The corresponding median RFS time was 3.3 months and 10.7 months, respectively, in patients with or without a TSC2 mutation. The difference approached near significance $(p=0.061)$ (Figure 3$)$. In the subgroup with BCLC stage B-C $(n=55)$, there were 8 patients with a TSC2 mutation and corresponding median RFS was 2.5 months. The median RFS of patients without a TSC2 mutation was 6.8 months. But the difference was not significant ( $p=0.118$ ) (Figure 4$)$.

\section{Discussion}

TSC2 was firstly identified in tuberous sclerosis complex in $1993 .^{12}$ Nowadays, it is known that TSC2 is a key regulator in the upstream signaling of $\mathrm{PI} 3 \mathrm{~K} / \mathrm{AKT} / \mathrm{mTOR}$ pathway, which plays an important role on HCC carcinogenesis and metastasis. ${ }^{13,14}$ Activation of the PI3K/AKT/ mTOR signaling pathway could induce many biological processes, which induced oncogenic transformation, such as accelerating cell proliferation, protecting cells against apoptosis, metabolic reprogramming, suppressing autophagy and senescence. ${ }^{15}$ As a downstream molecular of TSC2, mTORC1 was a key component in regulating a series of cancer-promoting biological processes by phosphorylation of proteins such as S6K1, 4E-BP1. ${ }^{16}$ The complex of TSC2 and TSC1 can inhibit mTORC1 and downstream signaling of PI3K/AKT/mTOR pathway. ${ }^{17,18}$ Therefore, TSC2 was an important negative regulator of $\mathrm{PI} 3 \mathrm{~K} / \mathrm{AKT} / \mathrm{mTOR}$ signaling pathway.

In our study, mutations of TSC2 were found in $12.6 \%$ of HCC patients. The mutation frequency of TSC2 was higher than previous reports, including 5\% from Schulze et $\mathrm{al}^{19}$ and 5\% from Totoki et $\mathrm{al}^{20}$ and 3.0\% to $4.5 \%$ from the cBioPortal (2019) database. This difference may be due to the background of different viral hepatitis. There were $88 \%$ of patients with $\mathrm{HBsAg}$ positive in this study, while the HBsAg positive only accounted for $14 \%$ and $23 \%$ in the study of Schulze et al $^{19}$ and Totoki et al. ${ }^{20}$ Our results showed that $T S C 2$ was the most commonly mutated gene of $\mathrm{PI} 3 \mathrm{~K} / \mathrm{AKT} / \mathrm{mTOR}$ signaling pathway. This is consistent with previous study of Ho and colleagues. ${ }^{21}$

As a negative regulator of the $\mathrm{PI} 3 \mathrm{~K} / \mathrm{AKT} / \mathrm{mTOR}$ pathway, low expression or loss of TSC2 implied overactivation of this pathway. It would inevitably lead to a series of biological processes conducive to the development of cancer. Some studies found that loss and mutations of TSC2 led to the loss function of TSC2 in HCC. ${ }^{11,21}$ In this study, we found that TSC2 mutations were significantly correlated with MVI and poorer Edmondson grade $(p<0.05)$. Similarly, a study reported TSC2 alterations were associated with HCC belonging to transcriptomic G3 subclasses characterized by poorly differentiation. ${ }^{22}$ This result indicated that TSC2 mutations were associated with poor biological characteristics of tumor in HCC patients. In patients with a TSC2 mutation, we found patients with MVI or maximum diameter of tumor 
Table 7 Univariate and Multivariate Cox Regression Analysis of Clinicopathological Characteristics and Gene Mutations with RFS of HCC Patients

\begin{tabular}{|c|c|c|c|c|c|c|}
\hline \multirow[t]{2}{*}{ Variables } & \multicolumn{3}{|c|}{ Univariate Analysis } & \multicolumn{3}{|c|}{ Multivariate Analysis } \\
\hline & HR & $95 \% \mathrm{Cl}$ & $\mathbf{P}$ & HR & $95 \% \mathrm{Cl}$ & $\mathbf{P}$ \\
\hline Age $(<65 / \geq 65)$ & $0.74 I$ & $0.430-1.274$ & 0.278 & & & \\
\hline Gender (male/female) & 0.674 & $0.349-1.303$ & $0.24 I$ & & & \\
\hline Hypertension (no/yes) & 0.474 & $0.263-0.855$ & 0.013 & - & - & 0.109 \\
\hline Diabetes (no/yes) & 0.642 & $0.296-1.390$ & $0.26 \mathrm{I}$ & & & \\
\hline Family history of cancer (no/yes) & 0.892 & $0.560-1.419$ & 0.629 & & & \\
\hline History of alcoholism (no/yes) & 0.881 & $0.564-1.376$ & 0.578 & & & \\
\hline $\mathrm{HBsAg}$ (negative/positive) & 1.939 & $0.895-4.200$ & 0.093 & & & \\
\hline HBV-DNA $(<|E+003 / \geq| E+0031 \mathrm{U} / \mathrm{mL})$ & 1.090 & $0.644-1.846$ & 0.749 & & & \\
\hline Anti-hepatitis virus treatment (no/yes) & 0.937 & $0.611-1.436$ & 0.765 & & & \\
\hline $\operatorname{AFP}(<400 / \geq 400 \mu \mathrm{g} / \mathrm{L})$ & 2.274 & I.478-3.498 & 0.000 & 1.715 & $1.093-2.693$ & 0.019 \\
\hline BCLC (0-A/B-C) & 3.513 & $2.290-5.388$ & 0.000 & 3.070 & $1.97 \mid-4.783$ & 0.000 \\
\hline Liver fibrosis (SIS2/S3S4) & 1.162 & $0.709-1.903$ & 0.551 & & & \\
\hline Edmondson grade (I-II/III-IV) & 1.542 & $1.009-2.358$ & 0.046 & - & - & 0.807 \\
\hline MVI (no/yes) & 1.947 & $1.264-2.998$ & 0.002 & - & - & 0.483 \\
\hline TP53 (Wild type/Mutant) & 0.948 & $0.622-1.444$ & 0.804 & & & \\
\hline TERT (Wild type/Mutant) & 1.093 & $0.713-1.675$ & 0.685 & & & \\
\hline CTNNBI (Wild type/Mutant) & 1.209 & $0.755-1.936$ & 0.430 & & & \\
\hline AXINI (Wild type/Mutant) & 0.914 & $0.497-1.68 \mid$ & 0.772 & & & \\
\hline TSC2 (Wild type/Mutant) & 2.043 & I.169-3.570 & 0.012 & $1.86 \mathrm{I}$ & $1.06 \mathrm{I}-3.267$ & 0.030 \\
\hline TSCI (Wild type/Mutant) & 1.405 & $0.444-4.447$ & 0.563 & & & \\
\hline TMB (<8.5/ $\geq 8.5$ mutations/Mb) & 0.685 & $0.412-1.139$ & 0.145 & & & \\
\hline
\end{tabular}

$>5 \mathrm{~cm}$ had higher TSC2 VAF than others $(p<0.05)$. This result indicated that high mutation load of TSC2 might correlate with poor biological characteristics of HCC.

We observed co-mutation between TSC2 mutations and TP53 mutations in the current study. TP53 gene is a key regulator in $T P 53 /$ cell-cycle pathway and its mutations are major drivers of HCC. ${ }^{23,24}$ TP53/cell-cycle pathway also plays a role in the occurrence and development of liver cancer. Previous studies have suggested possible associations between different genes. For example, Huang et $\mathrm{al}^{25}$ found the association between TSC2 and GSK3 beta expression. Peng et $\mathrm{al}^{26}$ found different combinations between TP53 polymorphisms and MDM2 polymorphisms were significantly correlated with the risk of HCC development. Our study found a higher proportion of TSC2 mutations in TP53 mutated HCC patients, indicating the potential correlation between them, which has not been reported before. Although Ho et $\mathrm{al}^{21}$ did not identify the correlation of TSC2 mutations and TP53 mutations in 95 patients with HBV-related HCC, the comutation of TSC2 and TP53 was still worthy of further investigation.

In this study, our results showed that the BCLC B-C stage, TSC2 mutations and preoperative serum AFP $\geq 400 \mu \mathrm{g} / \mathrm{L}$ were independent risk factors for poor RFS of HCC patients after hepatectomy. We did not find the correlation between these factors. It has been a consensus that BCLC staging and serum AFP level are extremely 


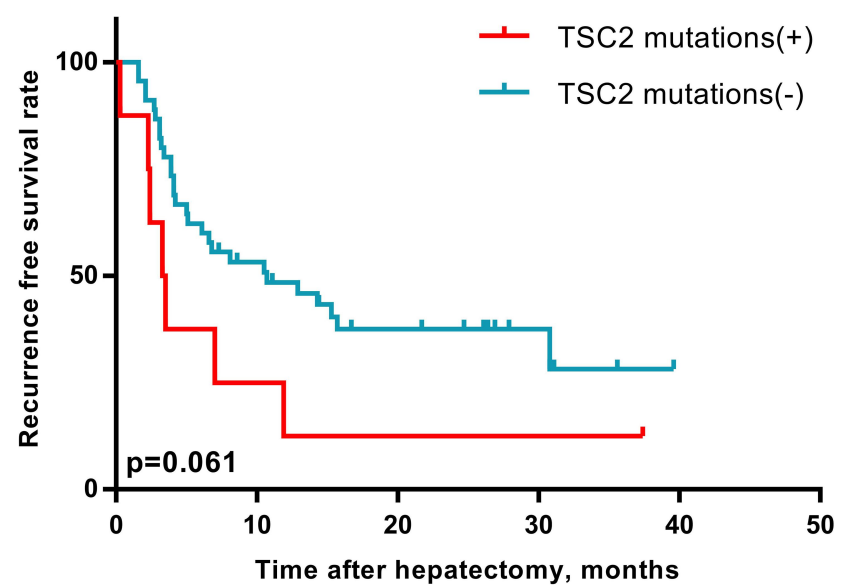

Figure 3 Survival curves of RFS according to TSC2 mutational status evaluated by Kaplan-Meier method in patients with preoperative serum AFP level above $400 \mu \mathrm{g} / \mathrm{L}$ $(n=53)$. Median RFS between patients with a TSC2 mutation and patients without TSC2 mutation was 3.3 vs 10.7 months $(p=0.061)$.

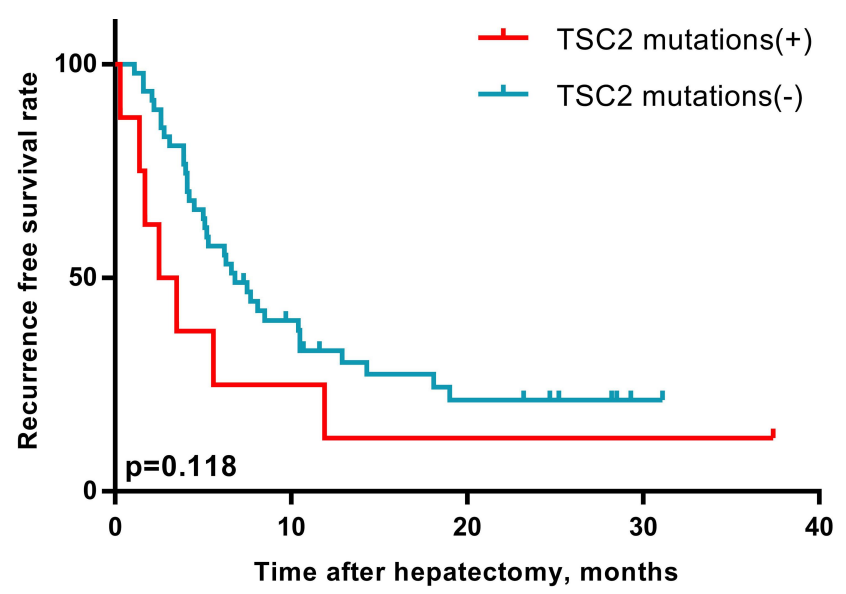

Figure 4 Survival curves of RFS according to TSC2 mutational status evaluated by Kaplan-Meier method in patients with BCLC stage B-C $(n=55)$. Median RFS between patients with a TSC2 mutation and patients without TSC2 mutation was 2.5 vs 6.8 months $(p=0.118)$.

valuable for the prognostic evaluation of $\mathrm{HCC}$ patients. We found that patients with a TSC2 mutation exhibited shorter RFS time after hepatectomy than those without a TSC2 mutation. The mutation of TSC2 was one of independent risk factors for both $\mathrm{HCC}$ recurrence within 1 year and poor RFS time after hepatectomy. This result showed that TSC2 mutations in HCC tissue might be one of the early recurrence factors for HCC patients underwent liver resection. Huang et $\mathrm{al}^{25}$ also reported that low expression of TSC2 was associated with poor prognosis of HCC patients. The mutation of TSC2 means the dysfunction of TSC2 and may promote the invasion and aggression of $\mathrm{HCC}$, supporting that TSC2 could predict early recurrence of $\mathrm{HCC}$ patients. In subgroup with AFP level above $400 \mu \mathrm{g} / \mathrm{L}$, the median RFS was shorter in patients with a TSC2 mutation than those without TSC2 mutation, and the difference was trends clinically significant. The TSC2 mutations may predict poorer RFS for HCC patients with AFP level above $400 \mu \mathrm{g} / \mathrm{L}$. We inferred that the HCC patients with TSC2 mutation might be a group at high risk of early recurrence after hepatectomy. For these patients, surveillance was more important for detecting recurrence and early intervention.

This study has some limitations. Firstly, this is a monocenter study and lacks representativeness. Secondly, the follow-up time of this study was relatively shorter. Thus, multicenter study is necessary to enrich the results. We expect that we can acquire more convincing results with the extension of follow-up time and the increase of sample size.

\section{Conclusion}

In conclusion, TSC2 mutations were significantly associated with MVI in liver para-carcinoma tissue and Edmondson grade III-IV in patients with HCC and were independently associated with recurrence within 1 year and poorer RFS after hepatectomy. The TSC2 mutation may be a potential predictor for early recurrence in $\mathrm{HCC}$ patients underwent hepatectomy.

\section{Ethics Approval and Consent}

All procedures performed in studies involving human participants were in accordance with the ethical standards of the institutional and/or national research committee and with the 1964 Helsinki Declaration and its later amendments or comparable ethical standards. The study was approved by the Ethics Committee of the Affiliated Hospital of Qingdao University (ethics approval number: QDFYKYLLL-20161212). Informed consent was obtained from all patients included in our study. All participants and contributors of this study have signed informed consent for publication.

\section{Acknowledgment}

Thanks to OrigiMed for the technical support with NGS.

\section{Author Contributions}

All authors made a significant contribution to the work reported, whether that is in the conception, study design, execution, acquisition of data, analysis and interpretation, or in all these areas; took part in drafting, revising or critically reviewing the article; gave final approval of the 
version to be published; have agreed on the journal to which the article has been submitted; and agree to be accountable for all aspects of the work.

\section{Funding}

This study did not receive any funding.

\section{Disclosure}

All authors declare no conflicts of interest in this work.

\section{References}

1. Bray F, Ferlay J, Soerjomataram I, Siegel RL, Torre LA, Jemal A. Global cancer statistics 2018: GLOBOCAN estimates of incidence and mortality worldwide for 36 cancers in 185 countries. CA Cancer J Clin. 2018;68(6):394-424. doi:10.3322/caac.21492

2. Mazzola A, Costantino A, Petta S, et al. Recurrence of hepatocellular carcinoma after liver transplantation: an update. Future Oncol. 2015;11(21):2923-2936. doi:10.2217/fon.15.239

3. Kumar AM, Fredman ET, Coppa C, El-Gazzaz G, Aucejo FN, AbdelWahab M. Patterns of cancer recurrence in localized resected hepatocellular carcinoma. Hepatobiliary Pancreat Dis Int. 2015;14 (3):269-275. doi:10.1016/s1499-3872(15)60382-4

4. Huang J, Manning BD. The TSC1-TSC2 complex: a molecular switchboard controlling cell growth. Biochem J. 2008;412 (2):179-190. doi:10.1042/bj20080281

5. Mehta MS, Vazquez A, Kulkarni DA, et al. Polymorphic variants in TSC1 and TSC2 and their association with breast cancer phenotypes. Breast Cancer Res Treat. 2011;125(3):861-868. doi:10.1007/s10549010-1062-1

6. Chakraborty S, Mohiyuddin SM, Gopinath KS, Kumar A. Involvement of TSC genes and differential expression of other members of the mTOR signaling pathway in oral squamous cell carcinoma. BMC Cancer. 2008;8:163. doi:10.1186/1471-2407-8-163

7. Geng H, Li S, Guo Y, et al. Survival prediction for patients with lung adenocarcinoma: A prognostic risk model based on gene mutations. Cancer Biomark. 2020;27(4):525-532. doi:10.3233/cbm-191204

8. Lee YH, Do SK, Lee SY, et al. TSC2 genetic variant and prognosis in non-small cell lung cancer after curative surgery. Thorac Cancer. 2019;10(2):335-340. doi:10.1111/1759-7714.12951

9. Zhang Y, Jia QA, Kadel D, Zhang XF, Zhang QB. Targeting mTORC1/2 complexes inhibit tumorigenesis and enhance sensitivity to 5-fluorouracil (5-FU) in hepatocellular carcinoma: a preclinical study of mTORC1/2-targeted therapy in hepatocellular carcinoma (HCC). Med Sci Monit. 2018;24:2735-2743. doi:10.12659/ msm. 907514

10. Cho J, Lee J, Kim J, et al. Loss of tuberous sclerosis complex 2 (TSC2) as a predictive biomarker of response to mTOR inhibitor treatment in patients with hepatocellular carcinoma. Transl Oncol. 2016;9(5):466-471. doi:10.1016/j.tranon.2016.08.009

11. Huynh H, Hao HX, Chan SL, et al. Loss of tuberous sclerosis complex 2 (TSC2) is frequent in hepatocellular carcinoma and predicts response to mTORC1 inhibitor everolimus. Mol Cancer Ther. 2015;14(5):1224-1235. doi:10.1158/1535-7163.mct-14-0768
12. European Chromosome 16 Tuberous Sclerosis Consortium. Identification and characterization of the tuberous sclerosis gene on chromosome 16. Cell. 1993;75(7):1305-1315. doi:10.1016/00928674(93)90618-z.

13. Dimri M, Satyanarayana A. Molecular signaling pathways and therapeutic targets in hepatocellular carcinoma. Cancers (Basel). 2020;12 (2):491. doi:10.3390/cancers 12020491

14. Couri T, Pillai A. Goals and targets for personalized therapy for HCC. Hepatol Int. 2019;13(2):125-137. doi:10.1007/s12072-0189919-1

15. Aoki M, Fujishita T. Oncogenic roles of the PI3K/AKT/mTOR axis. Curr Top Microbiol Immunol. 2017;407:153-189. doi:10.1007/ 82_2017_6

16. Laplante M, Sabatini DM. mTOR signaling in growth control and disease. Cell. 2012;149(2):274-293. doi:10.1016/j.cell.2012.03.017

17. Ashworth RE, Wu J. Mammalian target of rapamycin inhibition in hepatocellular carcinoma. World J Hepatol. 2014;6(11):776-782. doi:10.4254/wjh.v6.i11.776

18. Tee AR, Fingar DC, Manning BD, Kwiatkowski DJ, Cantley LC, Blenis J. Tuberous sclerosis complex-1 and -2 gene products function together to inhibit mammalian target of rapamycin (mTOR)mediated downstream signaling. Proc Natl Acad Sci U S A. 2002;99(21):13571-13576. doi:10.1073/pnas.202476899

19. Schulze K, Imbeaud S, Letouzé E, et al. Exome sequencing of hepatocellular carcinomas identifies new mutational signatures and potential therapeutic targets. Nat Genet. 2015;47(5):505-511. doi:10.1038/ng.3252

20. Totoki Y, Tatsuno K, Covington KR, et al. Trans-ancestry mutational landscape of hepatocellular carcinoma genomes. Nat Genet. 2014;46 (12):1267-1273. doi:10.1038/ng.3126

21. Ho DWH, Chan LK, Chiu YT, et al. TSC1/2 mutations define a molecular subset of HCC with aggressive behaviour and treatment implication. Gut. 2017;66(8):1496-1506. doi:10.1136/gutjnl-2016312734

22. Calderaro J, Couchy G, Imbeaud S, et al. Histological subtypes of hepatocellular carcinoma are related to gene mutations and molecular tumour classification. $J$ Hepatol. 2017;67(4):727-738. doi:10.1016/j. jhep.2017.05.014

23. Zheng Y, Lv P, Wang S, Cai Q, Zhang B, Huo F. LncRNA PLAC2 upregulates p53 to induce hepatocellular carcinoma cell apoptosis. Gene. 2019;712:143944. doi:10.1016/j.gene.2019.143944

24. Bykov VJN, Eriksson SE, Bianchi J, Wiman KG. Targeting mutant p53 for efficient cancer therapy. Nat Rev Cancer. 2018;18(2):89-102. doi:10.1038/nrc.2017.109

25. Huang KT, Huang YH, Li P, et al. Correlation between tuberous sclerosis complex 2 and glycogen synthase kinase 3 beta levels, and outcomes of patients with hepatocellular carcinoma treated by hepatectomy. Hepatol Res. 2014;44(11):1142-1150. doi:10.1111/ hepr.12256

26. Peng Q, Lao X, Chen Z, et al. TP53 and MDM2 gene polymorphisms, gene-gene interaction, and hepatocellular carcinoma risk: evidence from an updated meta-analysis. PLoS One. 2013;8(12):e82773. doi:10.1371/journal.pone. 0082773 


\section{Publish your work in this journal}

Pharmacogenomics and Personalized Medicine is an international, peer-reviewed, open access journal characterizing the influence of genotype on pharmacology leading to the development of personalized treatment programs and individualized drug selection for improved safety, efficacy and sustainability. This journal is indexed on the American Chemical Society's Chemical Abstracts Service (CAS). The manuscript management system is completely online and includes a very quick and fair peer-review system, which is all easy to use. Visit http://www.dovepress.com/testimonials.php to read real quotes from published authors. 